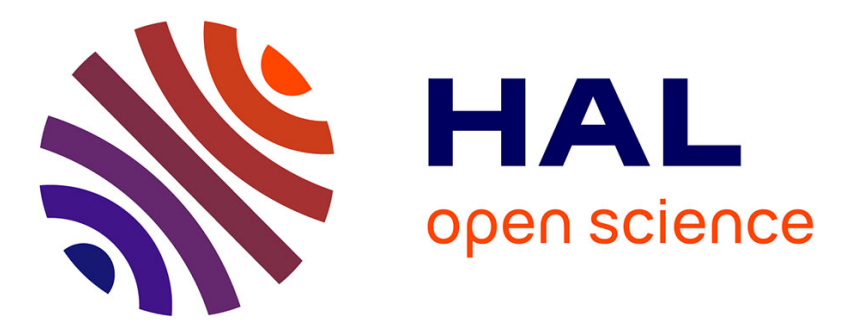

\title{
Calcul des propriétés énergétiques d'ions confinés dans une trappe quadrupolaire r.f. et refroidis par excitation d'une bande latérale
}

\author{
J. André, A. Teboul, F. Vedel
}

\section{- To cite this version:}

J. André, A. Teboul, F. Vedel. Calcul des propriétés énergétiques d'ions confinés dans une trappe quadrupolaire r.f. et refroidis par excitation d'une bande latérale. Journal de Physique Lettres, 1984, 45 (1), pp.1-10. 10.1051/jphyslet:019840045010100 . jpa-00232303

\section{HAL Id: jpa-00232303 https://hal.science/jpa-00232303}

Submitted on 1 Jan 1984

HAL is a multi-disciplinary open access archive for the deposit and dissemination of scientific research documents, whether they are published or not. The documents may come from teaching and research institutions in France or abroad, or from public or private research centers.
L'archive ouverte pluridisciplinaire HAL, est destinée au dépôt et à la diffusion de documents scientifiques de niveau recherche, publiés ou non, émanant des établissements d'enseignement et de recherche français ou étrangers, des laboratoires publics ou privés. 


\title{
LE JOURNAL DE PHYSIQUE-LETTRES
}

J. Physique - LETTRES 45 (1984) L-1 - L-10

1er JANVIER 1984, PAGE L- 1

Classification

Physics Abstracts

$07.75-05.40$

\section{Calcul des propriétés énergétiques d'ions confinés dans une trappe quadrupolaire r.f. et refroidis par excitation d'une bande latérale}

\author{
J. André, A. Teboul et F. Vedel \\ Physique des Interactions Ioniques et Moléculaires (*), \\ Université de Provence, Centre de St-Jérôme, 13397 Marseille Cedex 13, France
}

(Reçu le 4 août 1983, accepté le 14 novembre 1984)

\begin{abstract}
Résumé. - L'article décrit un formalisme semi-classique en vue de l'étude des propriétés énergétiques d'ions confinés dans une trappe quadrupolaire r.f. et refroidis par excitation d'une bande latérale. Ce formalisme est appliqué à un modèle de trappe unidimensionnel et testé sur des ions $\mathrm{Mg}^{+}$et $\mathrm{Be}^{+}$ confinés dans le domaine de l'approximation adiabatique. La température calculée des ions est de l'ordre du double de celle déduite d'un modèle de puits de potentiel harmonique. Les résultats obtenus par ce formalisme sont en accord avec ceux calculés à partir d'un formalisme quantique.
\end{abstract}

\begin{abstract}
A semi-classical formalism is described in order to compute the energetic properties of ions confined in a quadrupole r.f. trap and cooled by sideband excitation. This formalism is applied to an one-dimensional trap model and it is tested using $\mathrm{Mg}^{+}$and $\mathrm{Be}^{+}$ions confined within the adiabatic approximation domain. The computed ionic temperature is about twice greater than deduced from a harmonic potential well model. The results given by this formalism agree with those which are deduced from a quantum one.
\end{abstract}

\section{Introduction.}

L'utilisation des trappes quadrupolaires est souvent restreinte en raison de la température importante des ions. Ceci justifie les nombreux travaux sur les techniques de refroidissement. Dans le cas des ions lourds, la méthode de refroidissement par excitation d'une bande latérale (appelée également refroidissement par excitation laser) apparaît - au vu des résultats expérimentaux indiquant une température de l'ordre du $\mathrm{mK}$ - la plus efficace à ce jour [1-5]. Un certain nombre de travaux théoriques ont permis d'expliquer finement le processus de refroidissement et ont estimé la température limite des ions [5-8]. Dans le cas de la trappe quadru-

$\left(^{*}\right)$ Equipe de recherche associée au C.N.R.S. no 0898. 
polaire r.f., toutefois, la répartition du potentiel électrique de confinement est assimilée dans ces calculs à un puits de potentiel harmonique, ce qui peut amener des différences importantes entre la valeur prévue et la température limite vraie. L'étude de la vitesse de refroidissement, plus rarement abordée, a été également restreinte au modèle du puits de potentiel harmonique. Il faut, enfin, noter qu'aucune étude théorique ne tient quantitativement compte des perturbations apportées dans le processus de refroidissement par les collisions avec un gaz parasite ou par le phénomène de charge d'espace.

Dans plusieurs articles, nous avons développé un formalisme permettant l'étude des propriétés statistiques des ions produites par les collisions $[9,10]$. Nous adoptons ici ce formalisme pour calculer les propriétés énergétiques des ions confinés dans un modèle de trappe unidimensionnel quadrupolaire (qui s'est avéré être efficace pour des études analogues) et soumis au processus de refroidissement par excitation d'une bande latérale. Les degrés de liberté externes de l'ion sont traités classiquement. L'interaction de l'ion avec l'onde électromagnétique est calculée dans la limite des champs faibles ; cette technique est donc particulièrement adaptée à la description du comportement d'ions pour lesquels la transition active se situe dans l'ultra-violet, domaine où les sources d'excitation sont peu intenses.

\section{Formalisme.}

2.1 HYPOTHÈSES GÉNÉRALES. - Le mouvement d'un ion confiné dans une trappe quadrupolaire r.f. est déterminé par la fréquence du champ r.f. $(\Omega / 2 \pi)$, deux paramètres $(a, q)$ qui définissent le point de fonctionnement dans le domaine de stabilité et six variables $\left(u_{x}, u_{y}, u_{z}, y_{x}, y_{y}\right.$, $y_{z}$ ) qui caractérisent les conditions initiales et qui sont constantes en l'absence d'interaction. L'élongation dans une direction est à chaque instant donnée par :

$$
z_{i}(t)=\frac{u_{i}}{\omega_{i}}\left[A_{i}(t) \cos \left(\omega_{i} t-\phi_{i}\right)-B_{i}(t) \sin \left(\omega_{i} t-\phi_{i}\right)\right], \quad i=x, y, z
$$

Dans cette équation, les grandeurs $\omega_{i} / 2 \pi$ (fréquences séculaires) se déduisent de $\Omega / 2 \pi$ (fréquence micrométrique) par la relation suivante :

$$
\omega_{i}=\beta_{i} \Omega / 2
$$

où $\beta_{i}$ dépend de $(a, q) ; A_{i}(t)$ et $B_{i}(t)$ sont des fonctions périodiques de la période micrométrique, $T_{m}=2 \pi / \Omega$, dont la forme dépend de $(a, q)$. Le modèle unidimensionnel utilisé consiste à supposer que l'ion reste en mouvement le long de l'axe de symétrie $O z$ de la trappe et la seule équation du mouvement à considérer est la composante « $z$ » du système (1), que nous écrivons par la suite :

$$
z(t)=\frac{u}{\omega}[A(t) \cos (\omega t-\phi)-B(t) \sin (\omega t-\phi)]
$$

Nous supposons également que l'ion possède une transition de caractère dipolaire électrique, de fréquence $\omega_{0} / 2 \pi$ entre un état fondamental et un état excité, et une demi-largeur associée $\gamma$. L'ion interagit avec une onde électromagnétique monochromatique, de fréquence $\omega_{L} / 2 \pi$ légèrement inférieure à $\omega_{0} / 2 \pi$ et qui se propage parallèlement à la direction de son mouvement avec un vecteur d'onde $k_{L}$ supposé orienté positivement.

La technique de refroidissement utilise la différence entre l'énergie des photons absorbés et celle des photons émis spontanément au cours des interactions des ions avec le champ électromagnétique. Elle paraît ainsi d'autant plus efficace que la durée de vie du niveau excité est faible, ce qui se traduit par une largeur naturelle toujours très supérieure au $\mathbf{M H z}$, ordre de grandeur de la fréquence d'oscillation de l'ion dans la trappe. Cette dernière situation permet de faire l'hypothèse que l'ion reste en mouvement uniforme durant l'intervalle de temps caractéristique d'un cycle 
absorption d'un photon/émission d'un photon. L'ion est caractérisé par une impulsion $P_{i}=\hbar K_{i}$ avant l'interaction et $P_{f}=\hbar K_{f}$ après celle-ci. Soit $k_{d}$ (resp. $\omega_{d} / 2 \pi$ ) le vecteur d'onde (resp. la fréquence) du photon diffusé. Dans un modèle unidimensionnel, $k_{d}$ n'a que deux directions possibles et pour le processus de refroidissement, seules les interactions pour lesquelles $k_{d}$ est antiparallèle à $k_{L}$ sont actives. Leur densité d'interaction élémentaire à partir de la valeur $K_{i}$ est donnée par la formule de Heisenberg-Kramer suivante [6] :

$$
f\left(K_{i} \rightarrow K_{f}\right)=\frac{C}{\omega_{L} \omega_{d}} \frac{\delta\left(k_{L}+K_{i}-k_{d}-K_{f}\right) \delta\left(\omega_{d}-\omega_{L}+\frac{\hbar}{2 m}\left(K_{f}^{2}-K_{i}^{2}\right)\right)}{\left[\omega_{0}-\omega_{L}+\frac{\hbar}{2 m}\left(k_{i}+2 k_{L} K_{i}\right)\right]^{2}+\gamma^{2}},
$$

équations que nous réécrivons :

$$
\begin{aligned}
f\left(K_{i} \rightarrow K_{f}\right) & =g\left(K_{i}\right) \delta\left(k_{L}+K_{i}-k_{d}-K_{f}\right) \delta\left(\omega_{d}-\omega_{L}+\frac{\hbar}{2 m}\left(K_{f}^{2}-K_{i}^{2}\right)\right) \\
g\left(K_{i}\right) & =\frac{C}{\omega_{L} \omega_{d}} \frac{1}{\left[\omega_{0}-\omega_{L}+\frac{\hbar}{2 m}\left(k_{L}+2 k_{L} K_{i}\right)\right]^{2}+\gamma^{2}}
\end{aligned}
$$

Ainsi que nous le verrons plus loin, la constante $C$ où apparaît l'intensité du champ électromagnétique disparaît des équations qu'il faut résoudre.

Sous l'effet des interactions, les paramètres $u$ et $\phi$ changent de valeurs et définissent deux fonctions aléatoires $U(t)$ et $\phi(t)$. Ces interactions étant " quasi-instantanées », les propriétés générales de $U(t)$ et $\phi(t)$ ont déjà été étudiées dans la référence 9 et nous indiquons uniquement les résultats.

2.2 EQUATION GÉNÉRATRICE. - $\Phi(t)$ est assimilable à une fonction aléatoire uniformément répartie sur $(0,2 \pi)$ et indépendante de $U(t)$ si la densité d'interaction est supérieure à la fréquence d'oscillation de l'ion dans la trappe. Cette condition est respectée pour les ions possédant la transition active dans l'ultraviolet (ions $\mathrm{Mg}^{+}$ou $\mathrm{Be}^{+}$par exemple), cas auquel nous limitons l'étude présente. $U(t)$ est décrit par un processus de Markov à valeur dans $R^{+}$. Celui-ci n'est pas homogène à cause du champ radiofréquence qui oscille à la période $T_{m}$. Mais si on se limite à l'étude des propriétés de $U(t)$ pour la suite des instants $0, T_{m}, 2 T_{m}, \ldots, K T_{m}, \ldots$, le processus résultant est homogène. Il est entièrement caractérisé par une distribution de probabilités initiales $\Pi_{0}(u)$ et l'ensemble des probabilités de transition élémentaires notées :

$$
p\left(u_{f}, u_{i}\right) T_{m}=\operatorname{Prob}\left[U\left(k T_{m}\right)=u_{f}, \mathrm{~d} u_{f} / U\left((k-1) T_{m}\right)=u_{i}\right] .
$$

Les propriétés énergétiques asymptotiques des ions se calculent alors à partir de la distribution de probabilité asymptotique $\Pi(u)$ qui est solution de l'équation génératrice :

$$
-\int p(u, v) \Pi(v) \mathrm{d} v+\Pi(u) \int p(v, u) \mathrm{d} v=0 .
$$

La résolution de cette équation nécessite le calcul de l'ensemble des valeurs de $p(u, v)$ en relation avec la méthode de résolution choisie.

2.3 Modèle DE FoKKer-PlancK. - La solution de l'équation intégrale 6 ne.peut être calculée numériquement qu'à la suite de simplifications obtenues soit en discrétisant cette équation, soit en transformant sa structure. Nous avons choisi la deuxième possibilité en utilisant un modèle 
de Fokker-Planck que nous avons testé par ailleurs sur un modèle académique [8]. Cette méthode consiste à approcher $\Pi(v)$, dans l'équation 6 , par son développement limité au second ordre autour de $\Pi(u)$. Cette équation se transforme alors en l'équation différentielle suivante :

$\Pi(u)\left[\int p(u, v) \mathrm{d} v-\int p(v, u) \mathrm{d} v\right]+\Pi^{\prime}(u) \int(v-u) p(u, v) \mathrm{d} v+\Pi^{\prime \prime}(u) \int \frac{(v-u)^{2}}{2} p(u, v) \mathrm{d} v=0$

$u$ ayant la dimension d'une vitesse, l'énergie dépend du carré de cette grandeur et l'existence d'une loi de distribution énergétique bornée impose que $\Pi(0)=0 . \Pi(u)$ est alors définie à une constante multiplicative près. Une seconde condition, telle que :

$$
\int \Pi(u) \mathrm{d} u=1
$$

détermine éventuellement la constante.

2.4 CALCUl DES DENSitÉS D'INTERACTIONS. - Les densités d'interactions élémentaires $p(v, u)$ sont obtenues suivant une méthode analogue à celle développée dans la référence 9 et adaptée à l'étude présente. Soit $u$ l'état initial d'un ion et soit une valeur particulière du couple $(\phi, t)$. On exprime d'abord l'impulsion initiale $\hbar K_{i}$ de l'ion en fonction de $u, \phi, t$. On calcule ensuite quelles sont les valeurs de l'impulsion associées à un ion situé dans l'état $v$ (il y a deux valeurs notées $\hbar K^{-}$et $\hbar K^{+}$, ainsi que l'indique la figure 1). Puis on évalue la probabilité pour qu'une inter-

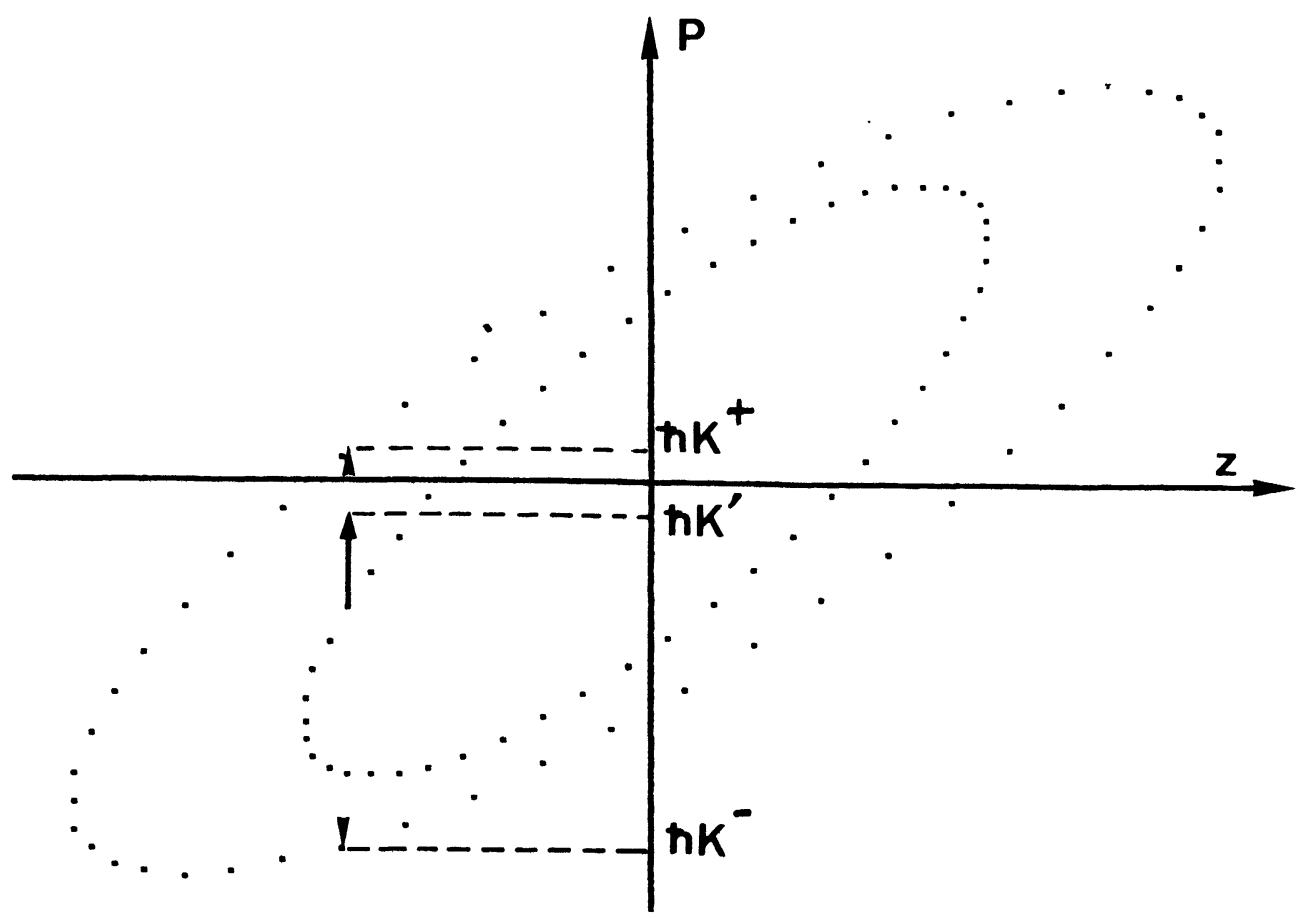

Fig. 1. - Représentation schématique du mouvement d'un ion dans l'espace des phases.

[Schematic representation of the ion motion in the phase space.] 
action conduise à une valeur de l'impulsion finale $\hbar K^{\prime}(u, \phi, t)$ comprise entre $\hbar K^{-}$et $\hbar K^{+}$. On somme enfin les probabilités ainsi obtenues pour chaque valeur de $(\phi, t)$ en les pondérant par la probabilité d'existence de ces valeurs $(\phi, t)$. On obtient alors la probabilité $P(v, u)$ pour qu'une interaction amène l'ion de l'état $u$ sur un état inférieur à $v$. L'expression de $p(v, u)$ se déduit de celle de $P(v, u)$ par dérivations :

$$
\begin{array}{r}
P(v, u)=\int_{0}^{v} p\left(u^{\prime}, u\right) \mathrm{d} u=\frac{1}{2 \Pi} \int_{0}^{T_{m}} \int_{0}^{2 \pi} g\left(K_{i}(u, \phi, t)\right)\left[H\left(K^{\prime}(u, \phi, t)-K^{-}(\phi, t, v)\right)-\right. \\
\left.-H\left(K^{\prime}(u, \phi, t)-K^{+}(\phi, t, v)\right)\right] \mathrm{d} \phi \mathrm{d} t
\end{array}
$$

$p(v, u)=\frac{\partial}{\partial v} P(v, u)$

Dans cette expression, $H$ représente la fonction échelon unité, et s'exprime en fonction de $u, \phi, t$. $g$ est définie en (4) suivant la relation :

$$
g\left(K_{i}(u, \phi, t)\right)=\frac{C}{\omega_{L} \omega_{d}} \frac{1}{\left[\omega_{0}-\omega_{L}+\frac{\hbar k_{L}^{2}}{2 m}+\frac{\hbar k_{L}}{m} K_{i}(u, \phi, t)\right]^{2}+\gamma^{2}} .
$$

L'existence de fonctions échelon unité dans l'intégrale de (8) rend son calcul numérique malaisé par les techniques classiques et nous utilisons une méthode de Monte-Carlo développée dans la référence 10. Faisons le changement de variable $\lambda_{1}=\Omega t, \lambda_{2}=\omega t-\phi$ et posons $u^{\prime}(u, \phi, t)$ l'état final qui correspond à l'impulsion finale $\hbar K^{\prime}$ de l'ion. L'expression 8 se met sous la forme d'une espérance mathématique :

$$
p(v, u)=\frac{\partial}{\partial v} E\left[g\left(K_{i}\left(u, \Lambda_{1}, \Lambda_{2}\right)\right) H\left(u^{\prime}\left(u, \Lambda_{1}, \Lambda_{2}\right)-v\right)\right]
$$

où $\Lambda_{1}, \Lambda_{2}$ sont des variables aléatoires indépendantes uniformément réparties sur $(0,2 \pi)$. Cette dernière expression est approchée par :

$$
\begin{aligned}
p(v, u) \# \frac{1}{n \Delta v} \sum_{j=1}^{n} g\left(K_{i}\left(u, \lambda_{1 j}, \lambda_{2 j}\right)\right)\left[H\left(u^{\prime}\left(u, \lambda_{1 j}, \lambda_{2 j}\right)-v+\frac{\Delta v}{2}\right)-\right. & \left.-H\left(u^{\prime}\left(u, \lambda_{1 j}, \lambda_{2 j}\right)-v-\frac{\Delta v}{2}\right)\right]
\end{aligned}
$$

où $\Delta v$ est un élément d'intervalle très inférieur au support de $p, \lambda_{1 j}$ et $\lambda_{2 j}$ sont des échantillons tirés suivant les lois de $\Lambda_{1}$ et $\Lambda_{2}$.

\section{Résultats.}

3.1 LOI DE DISTRIBUTION DES VITESSES. - Nous avons étudié numériquement les propriétés énergétiques limites dans le cas de l'approximation adiabatique. Les équations du mouvement sont alors simplifiées et s'écrivent :

$$
\left.\begin{array}{l}
z(t) \# \frac{u}{\omega} \cos (\omega t-\phi) \\
\dot{z}(t) \# u[\sqrt{2} \sin \Omega t \cos (\omega t-\phi)-\sin (\omega t-\phi)] .
\end{array}\right\}
$$


Deux cas ont été abordés :

- Cas 1 : masse de l'ion : 24 u.m.a., vecteur d'onde incident :

$$
22,4 \times 10^{6} \text { u.s.i., } 10^{7} \leqslant \gamma \leqslant 10^{8} \mathrm{rad} . \mathrm{s}^{-1} \text {. }
$$

- Cas 2 : masse de l'ion : 9 u.m.a., vecteur d'onde incident :

$$
20,1 \times 10^{6} \text { u.s.i., } 10^{7} \leqslant \gamma \leqslant 10^{8} \mathrm{rad} . \mathrm{s}^{-1} .
$$

Ces deux cas correspondent respectivement aux ions $\mathrm{Mg}^{+}$et $\mathrm{Be}^{+}$qui font l'objet d'études expérimentales.

Sur la figure 2 , nous avons tracé les valeurs $\Pi(u)$ déduites de l'intégration de l'équation 6 (cercles noirs). Nous avons également tracé (trait plein) le graphe de la fonction :

$$
\tilde{\Pi}(u)=\frac{u}{\sigma^{2}} \mathrm{e}^{-u^{2} / 2 \sigma^{2}}
$$

pour la valeur de $\sigma$ qui approche au mieux $\Pi(u)$ dans le domaine $(0,2 \sigma)$. Le bon accord entre $\Pi(u)$ et $\tilde{\Pi}(u)$ obtenu dans ce cas particulier est généralisable pour l'ensemble des valeurs calculées. Il montre que la loi de distribution $\Pi(u)$ est approchable par une expression du type (13). Une telle loi implique pour la vitesse des ions une loi de distribution gaussienne et permet d'utiliser - avec précaution - la notion de température. Celle-ci est reliée à la dispersion de la vitesse et à $\sigma$ par la relation :

$$
\bar{T}=\frac{m}{k_{\mathrm{B}}}\left\langle\dot{z}^{2}\right\rangle=\frac{2 m}{k_{\mathrm{B}}} \sigma^{2},
$$

où $k_{\mathrm{B}}$ est la constante de Boltzmann.

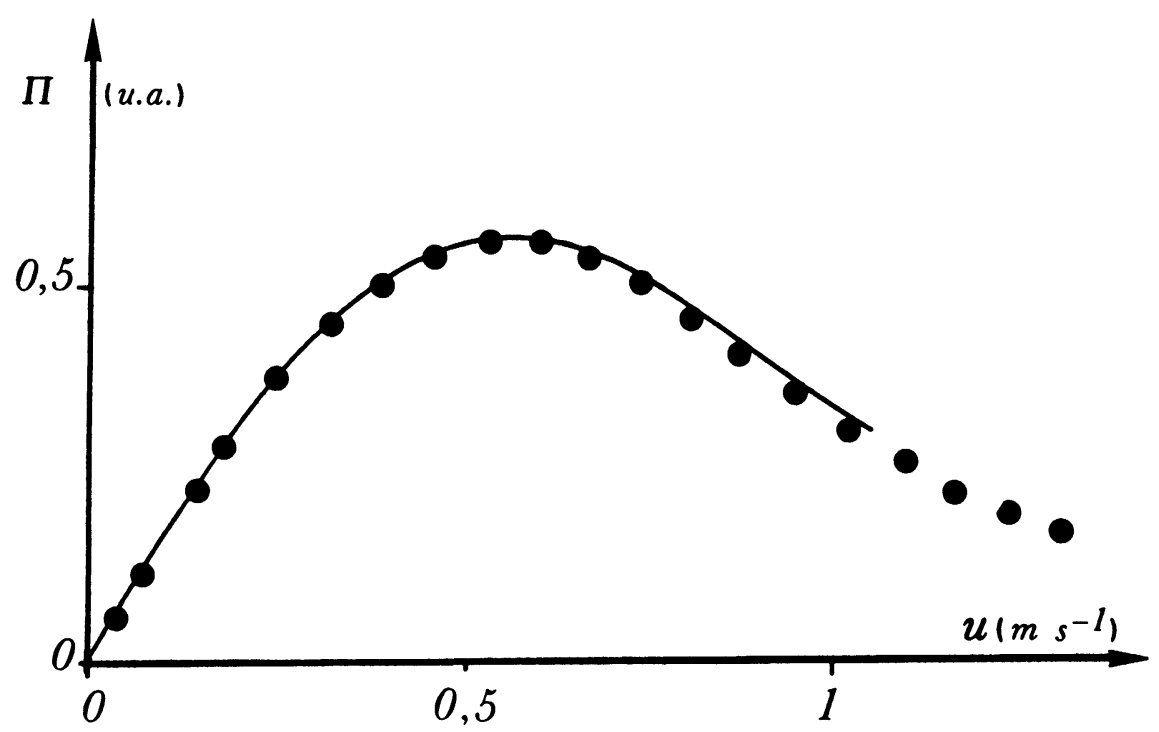

Fig. 2. - Evolution de $\Pi$ en fonction de $u$. Les cercles noirs représentent les valeurs calculées à partir du modèle de Fokker-Planck; la courbe représente la fonction $\left(u / \sigma^{2}\right) \exp \left(-u^{2} / 2 \sigma^{2}\right)$.

[Evolution of $\Pi$ versus $u$. Black circles represent computed values from the Fokker-Planck model; the curve represents the function $\left(u / \sigma^{2}\right) \exp \left(-u^{2} / 2 \sigma^{2}\right)$.] 
3.2 Température Des ions. - La figure 3 montre l'évolution de la température limite $\bar{T}$ des ions en fonction du décalage en fréquence $\Delta \omega=\omega_{0}-\omega_{L}$, pour différentes valeurs de $\gamma$. Les courbes notées $a$ (ion $\mathrm{Mg}^{+}$) et celles notées $b$ (ion $\mathrm{Be}^{+}$) sont semblables, ce qui suggère que les résultats obtenus ici sont éventuellement généralisables à d'autres espèces d'ions. Pour une valeur donnée de $\gamma$ la fonction $\bar{T}(\Delta \omega)$ passe par un minimum autour de la valeur $\Delta \omega \# \gamma$. Ce résultat est identique à celui qui avait été obtenu à partir d'un traitement quantique à l'aide d'un modèle de puits de potentiel harmonique [8]. Ces courbes sont dissymétriques. La température limite converge vers $\infty$ quand $\Delta \omega$ tend vers 0 , ce qui montre que $\gamma$ constitue une valeur inférieure du décalage pour l'obtention de faibles températures. Par contre $\Delta \omega$ peut être supérieur à $\gamma$ sans conséquence importante sur la valeur de $\bar{T}$.

Pour une valeur donnée de $\gamma$, la température minimale obéit à la relation :

$$
\bar{T}_{m}>\frac{2 \hbar \gamma}{k_{\mathrm{B}}}
$$

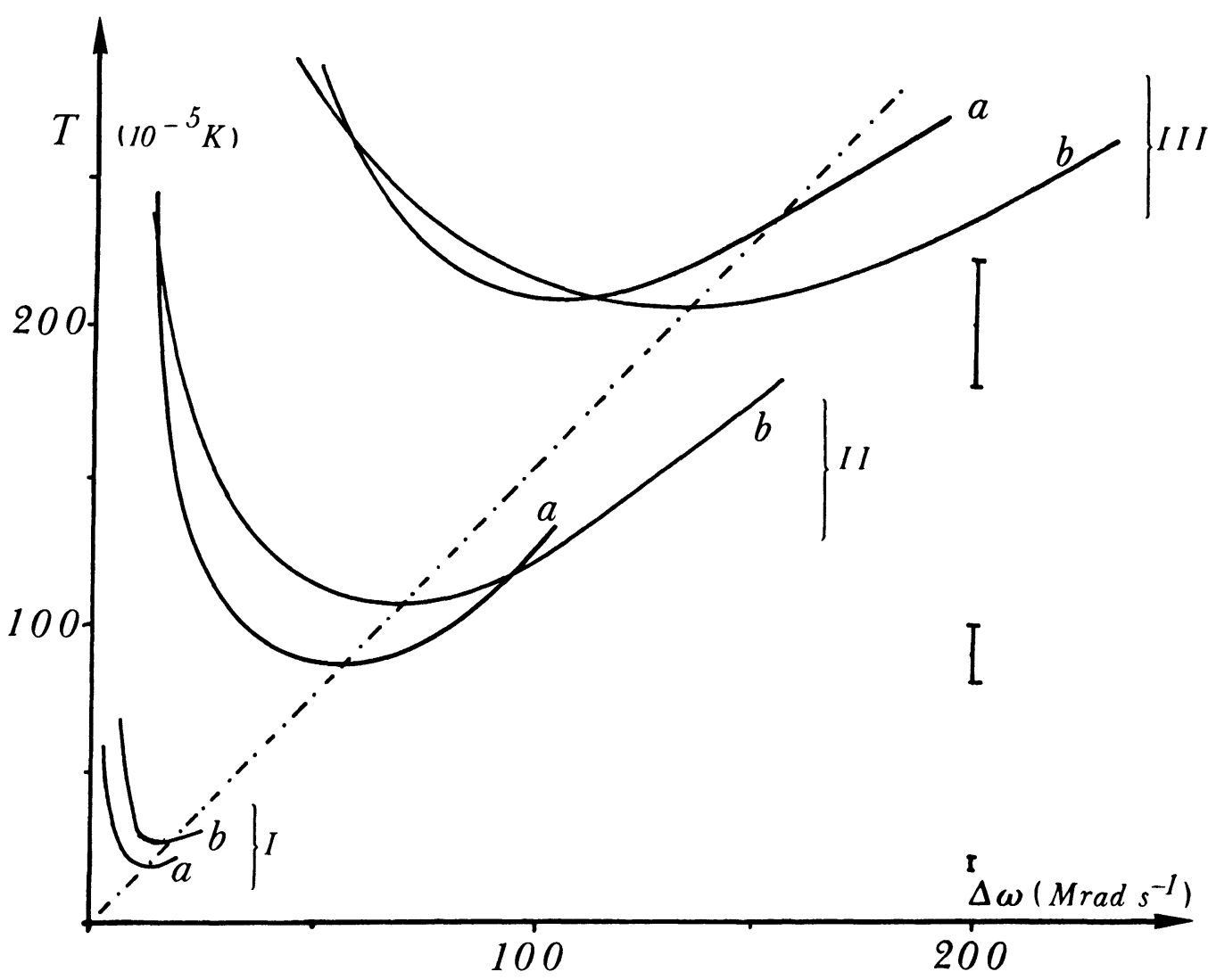

Fig. 3. - Influence de $\Delta \omega$ et $\gamma$ sur la température des ions. $a$ : ion $\mathrm{Mg}^{+} ; b:$ ion $\mathrm{Be}^{+} ; \mathrm{I}: \gamma=10 \mathrm{Mrad} . / \mathrm{s}$; II $: \gamma=50 \mathrm{Mrad}$./s; III $: \gamma=100 \mathrm{Mrad}$./s. Les barres d'erreur correspondent à deux fois l'écart-type; la droite en pointillés représente la fonction $2 \hbar \Delta \omega / k_{\mathrm{B}}\left(k_{\mathrm{B}}\right.$ est la constante de Boltzmann).

[Influence of $\Delta \omega$ and $\gamma$ upon the ionic temperature. $a$ : ion $\mathrm{Mg}^{+} ; b:$ ion $\mathrm{Be}^{+} ; \mathrm{I}: \gamma=10 \mathrm{Mrad} . / \mathrm{s} ; \mathrm{II}: \gamma=$ $50 \mathrm{Mrad} . / \mathrm{s}$; III $: \gamma=100 \mathrm{Mrad}$./s. The error bars correspond to twice the standard deviation; the dashed straight line corresponds to the function : $2 \hbar \Delta \omega / k_{\mathrm{B}}\left(k_{\mathrm{B}}\right.$ is the Boltzmann's constant).] 
Nous avons effectué une étude analogue sur l'ion $\mathrm{Mg}^{+}$en utilisant un modèle de puits de potentiel harmonique (voir Fig. 4). Les températures limites obtenues dans ce cas sont de l'ordre de $\hbar \gamma / k_{\mathrm{B}}$, en accord avec les prévisions théoriques antérieures. Le rapport des températures obtenues avec les différents modèles, de l'ordre de deux, montre que même dans le domaine de l'approximation adiabatique, le champ de confinement d'une trappe quadrupolaire r.f. ne peut être assimilé à un puits de potentiel harmonique.

3.3 COMPARAISON DU FORMALISME SEMI-CLASSIQUE ET DU FORMALISME QUANTIQUE. - Quel que soit le modèle de puits de confinement utilisé, les températures limites calculées sont toujours très faibles (inférieures à $10^{-3} \mathrm{~K}$ ). Dans un tel domaine, on peut se demander si le formalisme

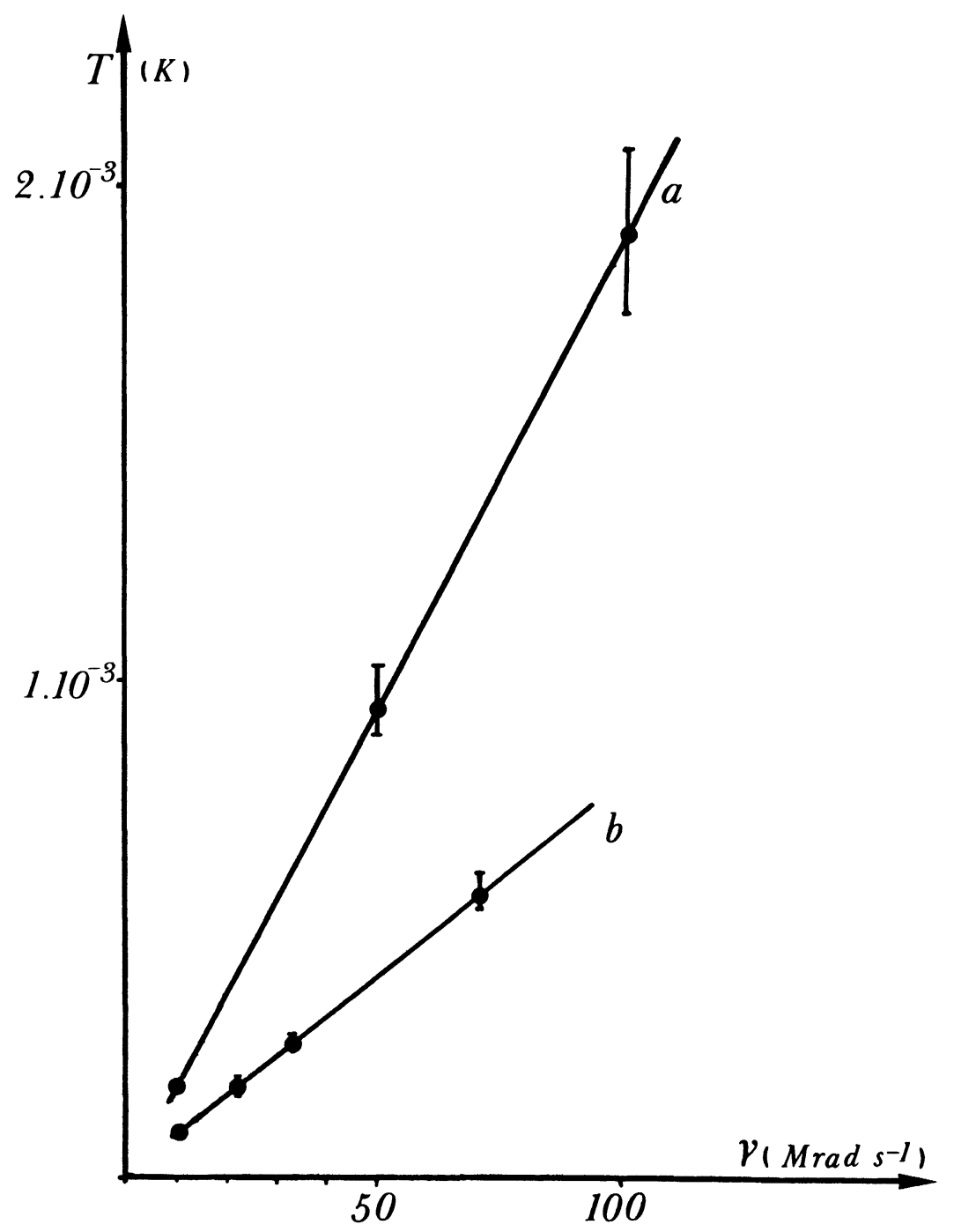

Fig. 4. - Comparaison entre le modèle de trappe unidimensionnel et le modèle du puits de potentiel harmonique. $a$ : modèle de trappe unidimensionnel; $b:$ modèle du puits de potentiel harmonique.

[Comparison of the one-dimensional trap model with the harmonic potential well model. $a$ : one-dimensional trap model ; $b$ : harmonic potential well model.] 
semi-classique reste valide. Celui-ci a donc été testé sur un modèle de puits de potentiel harmonique, pour un ensemble de valeurs des paramètres qui permettent une comparaison avec les résultats analogues obtenus à partir du formalisme quantique décrit précédemment [8]. Sur la figure 5 sont portées les températures limites minimales calculées pour le cas : masse de l'ion = 100 u.m.a., $k_{L}=12,8 \times 10^{6}$ u.s.i. Le bon accord entre les deux types de résultats montre que le modèle semi-classique fournit des prévisions réalistes dans ce domaine des températures atteintes par les ions refroidis par excitation d'une bande latérale.

\section{Conclusion.}

La méthode que nous présentons ici permet de calculer les propriétés énergétiques limites d'ions confinés dans une trappe quadrupolaire r.f. et refroidis par excitation d'une bande latérale. Cette méthode s'appuie sur un formalisme semi-classique dont nous avons testé le réalisme par comparaison avec un formalisme quantique développé précédemment; elle permet d'utiliser un modèle de trappe plus réaliste que le modèle du puits de potentiel harmonique, auquel les études précédentes se restreignaient. Dans le cadre de cet article, son application a été limitée au domaine de l'approximation adiabatique et à un modèle de trappe unidimensionnel. Qualitativement, les résultats obtenus ici sont similaires à ceux déduits d'autres travaux; ils présentent cependant des différences qui montrent les limites du modèle de puits de potentiel harmonique. En particulier, le rapport des températures est voisin de deux et pourrait être plus important en dehors du domaine de l'approximation adiabatique.

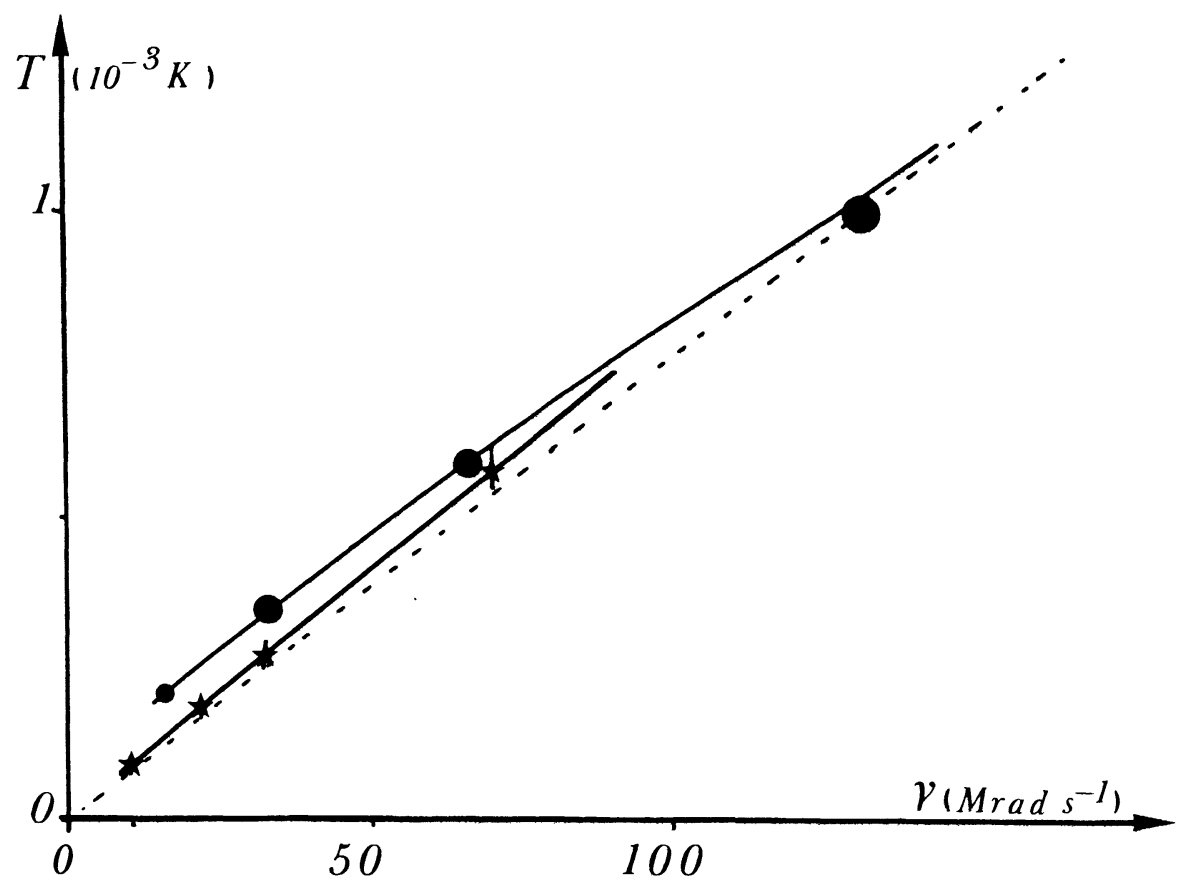

Fig. 5. - Comparaison entre le formalisme semi-classique et le formalisme quantique. Cercles noirs : valeurs calculées à partir du formalisme quantique de la référence 8; étoiles : valeurs calculées à partir du formalisme semi-classique.

[Comparison between the semi-classical formalism and the quantum formalism. Black circles : computed values from the quantum formalism described in reference 8; stars : computed values from the semi-classical formalism.] 
Un des intérêts du formalisme testé ici est sa faculté de s'appliquer à des conditions plus générales d'étude. Ainsi, il peut être adapté à des modèles de trappes tridimensionnels en remplaçant le modèle de Fokker-Planck par une méthode d'invariance temporelle que nous avons développée par ailleurs [11, 12]. Les températures et grandeurs énergétiques calculées ne peuvent être réalistes que dans un ensemble très restreint d'expériences où le milieu est extrêmement raréfié et où la population ionique ne dépasse pas quelques unités. En dehors de ces cas, on doit plus généralement admettre que les grandeurs sont fortement perturbées par les collisions éventuelles avec un gaz résiduel ou par les interactions ion-ion. Le présent formalisme est compatible avec ceux que nous avons exploités pour l'étude de l'influence des collisions élastiques et de l'effet de la charge d'espace faible [12-14]. Il paraît donc bien adapté à une description de l'ensemble des processus, utiles ou parasites, qui régissent le comportement des ions confinés dans une trappe quadrupolaire r.f...

\section{Remerciements}

Ce travail a été soutenu par la Direction de Recherches, Etudes et Techniques du Ministère de la Défense (Contrat no 83/1319).

\section{Bibliographie}

[1] Wineland, D. J., Dehmelt, H. G., Bull. Am. Phys. Soc. 20 (1975) 637.

[2] Neuhauser, W., Hohenstatt, M., Toschek, P., Dehmelt, H. G., Phys. Rev. A 22 (1980) 1137.

[3] Wineland, D. J., Itano, W. M., Phys. Lett. A 82 (1981) 75.

[4] Wineland, D. J., Itano, W. M., Bull. Am. Phys. Soc. 27 (1982) 471.

[5] Wineland, D. J., Itano, W. M., van Dyck, R. S. Jr, High resolution spectroscopy of stored ions, dans Advances in Atomic and Molecular Physics, vol. 19. Ed. Bederson and Bates (Academic Press) 1983 et références citées.

[6] Wineland, D. J., Itano, W. M., Phys. Rev. A 20 (1979) 1521.

[7] Javanainen, J., Stenholm, S., Appl. Phys. 21 (1980) 283.

[8] André, J., Teboul, A., Vedel, F., Vedel, M., J. Physique Colloq. 42 (1981) C8-315.

[9] ANDré, J., J. Physique 37 (1976) 719.

[10] ANDré, J., Vedel, F., J. Physique 38 (1977) 1381.

[11] André, J., Vedel, F., Vedel, M., J. Physique Lett. 24 (1979) L-633.

[12] Vedel, F., André, J., Vedel, M., Brincourt, G., Phys. Rev. A 27 (1983) 2321.

[13] Vedel, F., ANDré, J., Vedel, M., J. Physique 42 (1981) 1611.

[14] ANDRÉ, J., Vedel, F., Proceedings of the 15th E.G.A.S. conference, Madrid (1983). 